Article

\title{
Endoplasmic Reticulum Stress Signaling as a Therapeutic Target in Malignant Pleural Mesothelioma
}

\author{
Duo $\mathrm{Xu}^{1,2}$, Haitang Yang ${ }^{1,+}{ }^{1}$, Zhang Yang ${ }^{1,+}$, Sabina Berezowska ${ }^{3}{ }^{\circ}$, Yanyun Gao ${ }^{1}$, \\ Shun-Qing Liang ${ }^{1} \mathbb{D}$, Thomas M. Marti ${ }^{1} \mathbb{D}$, Sean R. R. Hall ${ }^{1}$, Patrick Dorn ${ }^{1}{ }^{\mathbb{D}}$, Gregor J. Kocher ${ }^{1}$, \\ Ralph A. Schmid ${ }^{1, *}$ and Ren-Wang Peng ${ }^{1, *}$ \\ 1 Department of General Thoracic Surgery, Department for BioMedical Research (DBMR), Inselspital, \\ Bern University Hospital, University of Bern, 3008 Bern, Switzerland; duo.xu@dbmr.unibe.ch (D.X.); \\ haitang.yang@dbmr.unibe.ch (H.Y.); zhang.yang@dbmr.unibe.ch (Z.Y.); yanyun.gao@dbmr.unibe.ch (Y.G.); \\ shun-qing.liang@umassmed.edu (S.-Q.L.); Thomas.Marti@insel.ch (T.M.M.); Sean.Hall@insel.ch (S.R.R.H.); \\ Patrick.Dorn@insel.ch (P.D.); Gregor.Kocher@insel.ch (G.J.K.) \\ 2 Graduate School for Cellular and Biomedical Sciences, University of Bern, 3008 Bern, Switzerland \\ 3 Institute of Pathology, University of Bern, 3008 Bern, Switzerland; sabina.berezowska@pathology.unibe.ch \\ * Correspondence: Ralph.Schmid@insel.ch (R.A.S.); Renwang.Peng@insel.ch (R.-W.P.) \\ + These authors contributed equally to this paper.
}

Received: 25 July 2019; Accepted: 22 September 2019; Published: 8 October 2019

check for updates

\begin{abstract}
Malignant pleural mesothelioma (MPM) is a lethal cancer with limited treatment options. No targeted therapy has emerged yet. Here, we performed an integrated molecular characterization of patient tumors in the TCGA dataset, and discovered that endoplasmic reticulum (ER) stress and the adaptive unfolded protein response (UPR) signaling are characteristically deregulated in MPM. Consequently, pharmacological perturbation of ER stress/UPR axis by HA15, an agent that induces persistent proteotoxic stress in the ER, selectively suppresses the viability of MPM cells including those refractory to standard chemotherapy. Mechanically, HA15 augments the already high basal level of ER stress in MPM cells, embarks pro-apoptotic malfunctional UPR and autophagy, which eventually induces cell death in MPM. Importantly, HA15 exerts anti-MPM effectiveness in a mouse model of patient-derived xenografts (PDX) without eliciting overt toxicity when compared to chemotherapy. Our results revealed that programs orchestrating ER stress/UPR signaling represent therapeutic vulnerabilities in MPM and validate HA15 as a promising agent to treat patients with MPM, naïve or resistant to chemotherapy.
\end{abstract}

Keywords: malignant pleural mesothelioma (MPM); endoplasmic reticulum (ER) stress; unfolded protein response (UPR); HA15; autophagy

\section{Introduction}

Malignant pleural mesothelioma (MPM) is a rare but aggressive cancer [1,2], consisting of epithelioid, mixed (biphasic) and sarcomatoid histological subtypes [3]. Despite progress in the understanding of the disease etiology, clinical management of MPM patients remains a significant but unmet challenge [4]. Whereas aggressive surgery is amenable for early-stage tumors [5], a majority $(80 \%)$ of patients with MPM are diagnosed at advanced stages, for which a dual chemotherapy regimen that combines cisplatin and pemetrexed is the only clinically approved therapy [6]. However, this systemic treatment only mildly improves patient survival (by three months only), as drug resistance, de novo, and/or acquired after the treatment, prevails [7]. 
Comprehensive genomic studies revealed that MPM is predominantly driven by loss of function mutations in tumor suppressor genes, most commonly the cyclin-dependent kinase inhibitor $2 \mathrm{~A} / 2 \mathrm{~B}$ gene $(C D K N 2 A / 2 B), B R C A 1$ associated protein 1 gene (BAP1), neurofibromin 2 gene (NF2), whereas therapeutically tractable oncogenic drivers are rare [8-10]. The lack of druggable activating mutations $[9,11,12]$ have significantly hampered the development of targeted therapies for MPM, which, however, suggests the importance of identifying and targeting functional cancer dependencies rather than specific driver mutations to combat MPM.

Cancer cells evolve in response to changes in oncogenic signaling and environmental pressures, for example, exacerbated secretory capacity, genomic instability and hypoxia $[13,14]$. Many stress-responsive mechanisms converge at an anabolic switch that increases protein metabolism, which induces proteotoxic endoplasmic reticulum (ER) stress, and in turn invokes the unfolded protein response (UPR) [15-17]. The UPR is mediated by three major signaling cascades initiated by so-called ER stress sensors: double-stranded RNA-activated protein kinase (PKR)-like ER kinase (PERK), inositol-requiring enzyme $1 \alpha$ (IRE1 $\alpha)$, and activating transcription factor 6 (ATF6). PERK, IRE1 $\alpha$, and ATF6 are ER membrane proteins that, at the steady state, are complexed with the chaperone protein glucose-regulated protein 78 (GRP78, also known as BiP). When threatened by increased protein-folding demand (ER stress), BiP is released, which activates PERK, IRE1 $\alpha$, and ATF6, and in turn, their downstream effectors to alleviate proteotoxic stress placed on the ER and to restore ER homoeostasis [18]. However, if the stress is irresolvable, the UPR signaling will trigger apoptosis/autophagy, leading to cell death $[19,20]$.

UPR dysfunction is implicated in many human diseases, for example, neurodegenerative, cardiovascular, and metabolic disorders. Hyperactive UPR has been reported in Alzheimer's disease (AD), progressive supranuclear palsy, and familial FTD (frontotemporal degeneration) with parkinsonism linked to chromosome 17 [21]. Recent studies have also uncovered that chronic ER stress is linked with endothelial dysfunction in cardiovascular diseases by increasing oxidative stress [22], and with insulin resistance and increased lipogenesis involved in metabolic disorders such as type II diabetes and obesity [23]. Deregulation of ER stress/UPR is also not uncommon in cancers [24,25] and many rely on the stress response signaling for survival or evasive resistance to cytotoxic therapeutics [26]. As such, targeting the UPR has emerged as a promising strategy for cancer therapy $[27,28]$.

A recent study by Cerezo et al., identified a new ER stress amplifier, termed HA15. HA15 binds to and inhibits the ATPase activity of BiP, thereby dissociating BiP from PERK, IRE1 $\alpha$, and ATF6 and triggering ER stress [29]. The anti-tumor effects of HA15 has been demonstrated in a variety of human cancers including melanoma, breast, pancreas, and adrenocortical carcinoma [29,30]. In this study, we showed that deregulation of ER stress/UPR signaling is a hallmark of MPM, which confers a specific vulnerability of therapeutic potential. Consequently, MPM cells, regardless of chemo-naïve or -resistant, are particularly susceptible to HA15. We further demonstrated that the antitumor effect of HA15 is attributable to its ability to induce excessive proteotoxic stress in the ER, which initiates malfunctional UPR, autophagy, and eventually the onset of apoptosis.

\section{Results}

\subsection{ER Stress and the Adaptive UPR Is Deregulated in Patients' MPM}

To unravel cellular pathways that may represent specific dependencies of MPM, we performed an integrated analysis of patients' MPM based on transcriptomic and clinical data available at the Cancer Genome Atlas (TCGA) [31]. Gene enrichment set analysis (GESA) revealed that genes involved in ER stress and the adaptive UPR (UPR gene signature) were significantly enriched in the patients' tumors compared to normal lung/pleura tissues (Figure 1A). Consistently, the UPR gene signature was positively correlated with proliferation and resistance to apoptosis, assessed by the expression of PCNA and BIRC5, encoding the proliferative marker proliferating cell nuclear antigen (PCNA) and anti-apoptotic protein SURVIVIN (also called baculoviral inhibitor of apoptosis repeat-containing 5 or 
BIRC5), respectively (Figure 1B). Notably, the UPR gene signature is of predictive value for patients with MPM, with an elevated UPR signature associated with poor prognosis (Figure 1C). Further examination of the transcriptomic data of patient-derived MPM [32] revealed that numerous genes involved in ER-related functions and/or proteostatic processes (i.e., FKBP14 [peptidyl-prolyl cis-trans isomerase (PPIase) FKBP14], SSR1 (translocon-associated protein subunit alpha or TRAP $\alpha$ ), HSP90B1 (heat shock protein 90 beta family member 1) and HSPA5/GRP78 (BiP)) are expressed at high levels in MPM compared to normal tissues (Figure 1D). Of particular interest, HSPA5/GRP78 and its protein product $\mathrm{BiP}$, a key ER chaperone and a master regulator of ER stress and the adaptive UPR, were generally overexpressed in MPM cells, except for $\mathrm{H} 28$, which had a lower BiP protein level than Met-5A (Figure 1E,F). Thus, deregulation of ER stress/UPR signaling is a characteristic feature for MPM and associated with poor prognosis in MPM patients. The original pictures of Western blotting can be found in Figure S1.

A

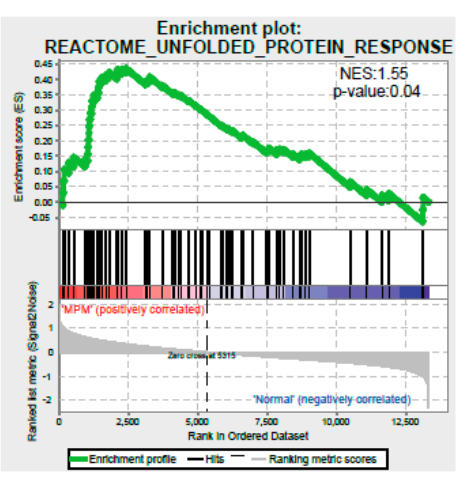

B
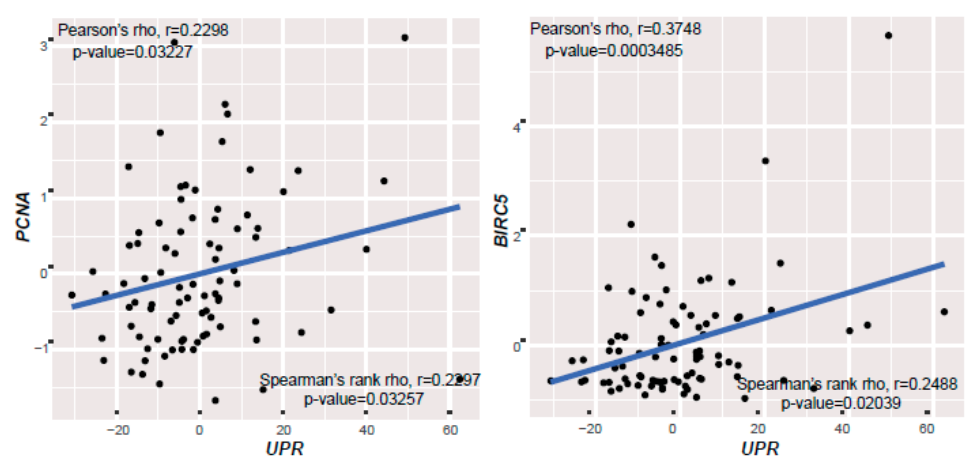

D

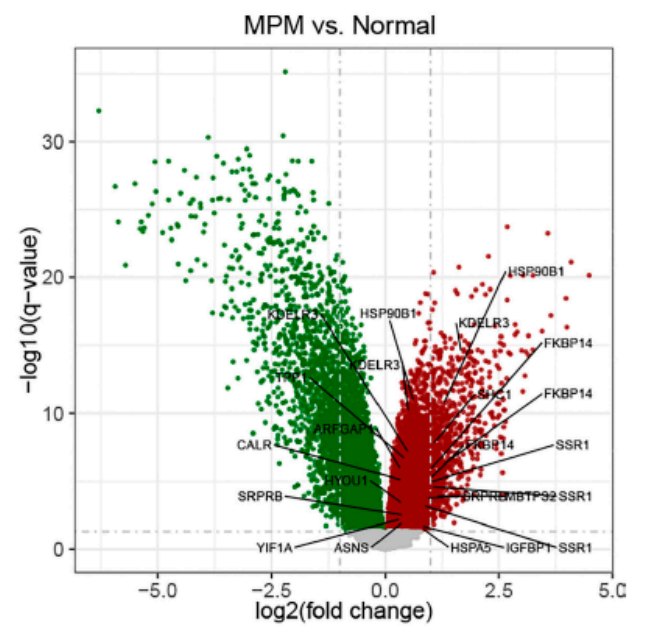

Figure 1. Cont. 
E

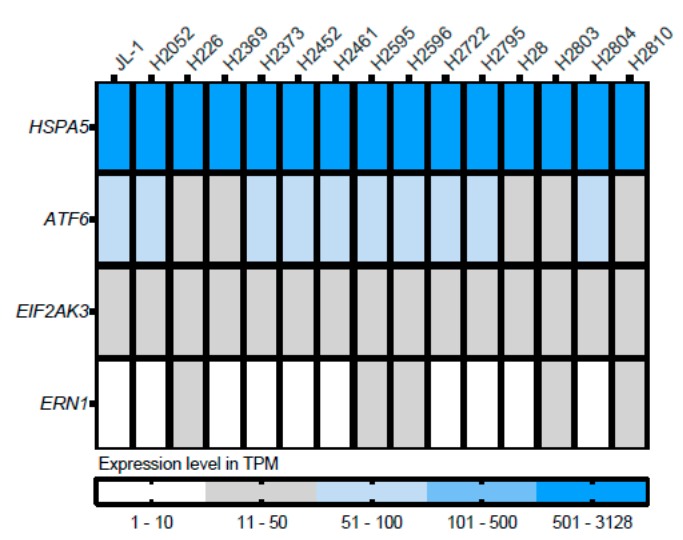

$\mathbf{F}$

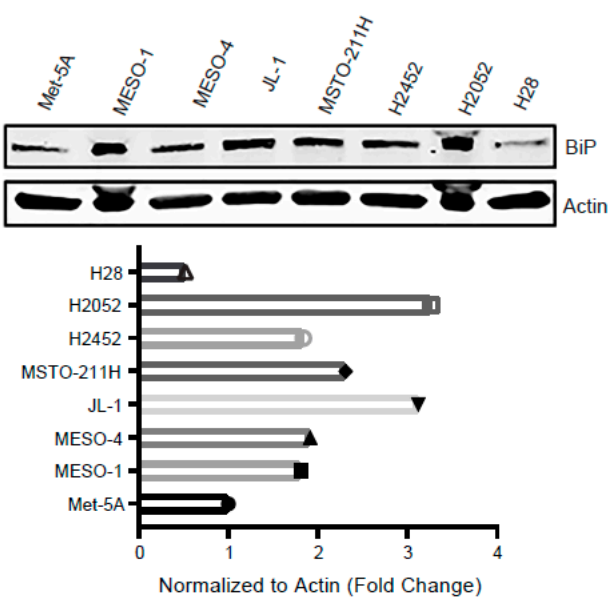

Figure 1. Endoplasmic reticulum (ER) stress/unfolded protein response (UPR) signaling is deregulated in malignant pleural mesothelioma (MPM). (A) Gene set enrichment analysis (GSEA) of the dataset (GSE2549) revealed significant enrichment of UPR in MPM tumor samples. (B) The UPR gene signature was positively correlated with the proliferative marker PCNA and anti-apoptotic marker SURVIVIN/BIRC5. Gene expression data of MPM were downloaded from TCGA, with Pearson/Spearman coefficients and $p$-value determined using R (version 3.4.3). (C) Kaplan-Meier analysis of a TCGA cohort of MPM patients $(n=86)$. The UPR gene signature was dichotomized based on the optimal cut-off value, and patient survival data were extracted for further analysis using $\mathrm{R}$ (Version 3.4.3). The $p$-value was calculated by the log-rank test. (D) The volcano plot of transcriptional comparison between the patients' MPM vs. normal lung tissues (GSE51024). (E) Heatmap of key UPR gene expression (TPM) in the MPM cell lines. The expression profile was obtained from the European Bioinformatics Institute of European Molecular Biology Laboratory (EMBL-EBI). (F) Immunoblots of $\mathrm{BiP}$ in normal mesothelial cells (Met-5A) and MPM cell lines. Relative expression of BiP is shown underneath, with signal intensity quantified by ImageJ and normalized to the loading control ( $\beta$-actin). The value in the Met-5A was set as 1 .

\subsection{Excessive ER Stress Induced by HA15 Selectively Impairs MPM Cells}

To address if ER stress/UPR signaling can be a potential therapeutic target in MPM, we treated a panel of cells including MPM cell lines across all histological subtypes (MESO-1, MESO-4, H28, JL-1, MSTO-211H, and H2052), a primary MPM culture (BE261T) and non-transformed normal cells (hFb16lu, Met-5A), with HA15, an agent inducing excess ER stress by specifically targeting BiP [29]. HA15 preferentially suppressed cell viability of MPM cells, with the $50 \%$ inhibitory concentration $\left(\mathrm{IC}_{50}\right)$ ranging from 5.71 to $20.51 \mu \mathrm{M}$, whereas it only mildly affected Met-5A and hFb16lu cells (Figure 2A,B). Clonogenic assay confirmed that HA15 is far more deleterious for MPM cells than fibroblast $\mathrm{hFb} 16 \mathrm{lu}$ and mesothelial Met-5A cells (Figure $2 \mathrm{C}-\mathrm{E}$ ). These results suggest that targeting BiP by HA15 preferentially inhibits MPM cells and is therefore a potential therapeutic strategy for MPM. 
A

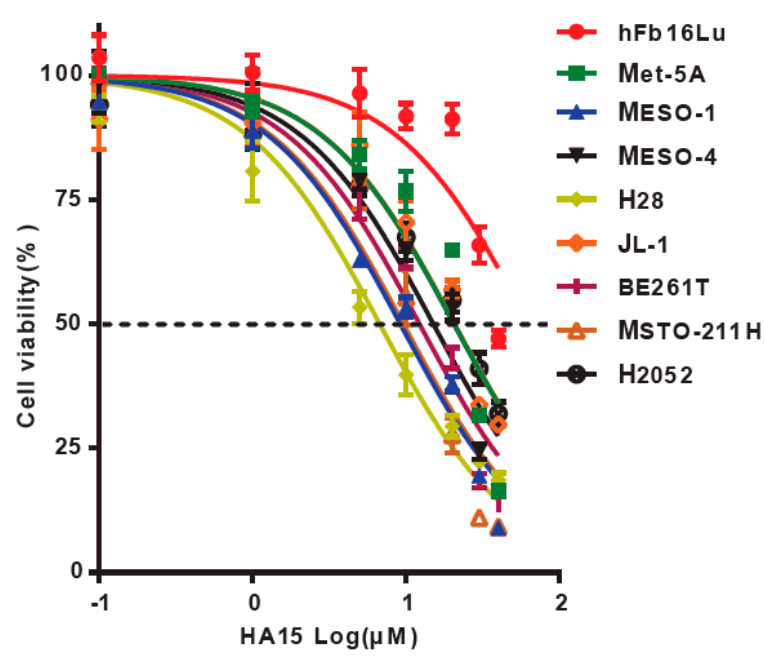

B

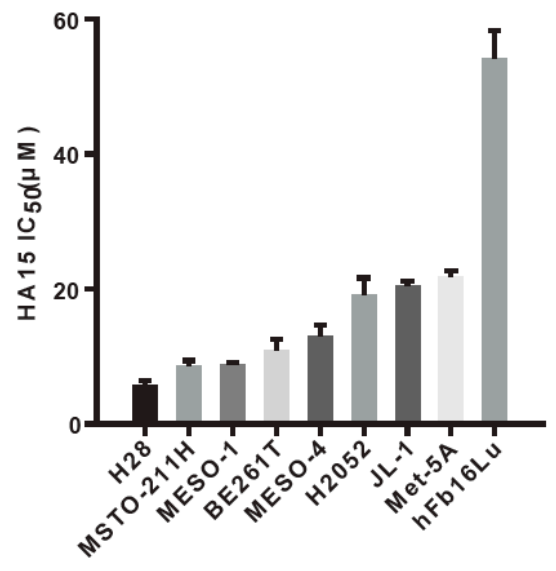

C

$\mathrm{HA} 15(\mu \mathrm{M})$
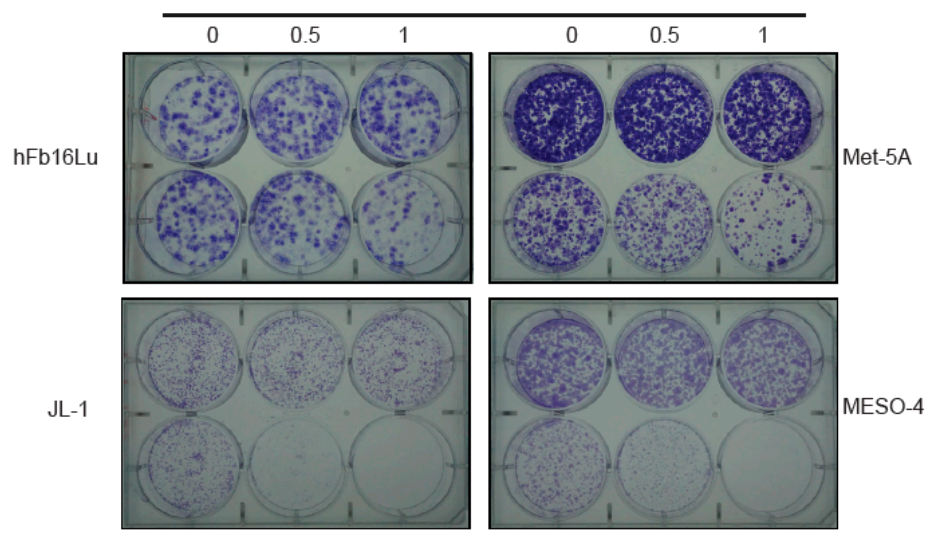

H28
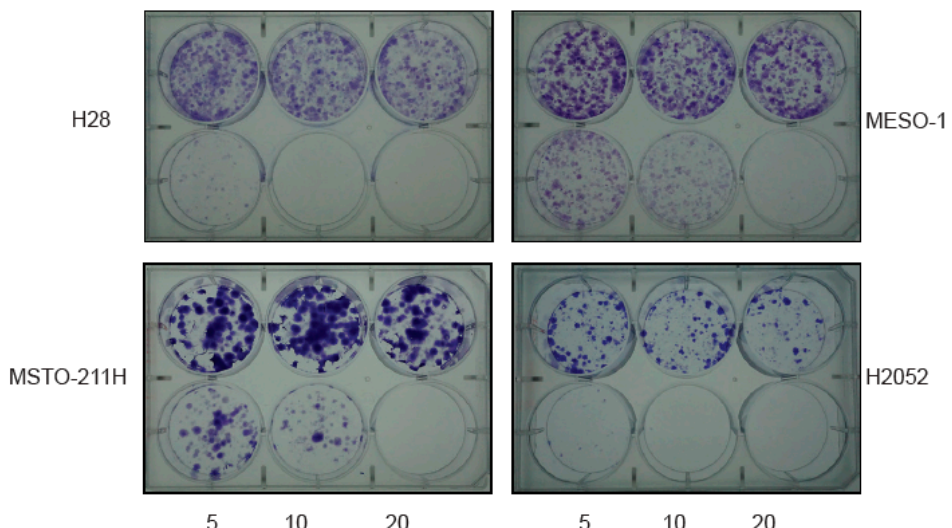

Figure 2. Cont. 
D

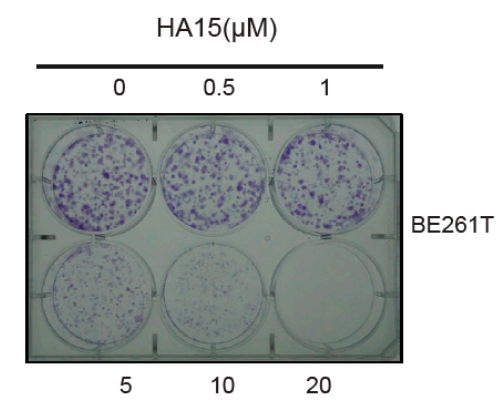

E

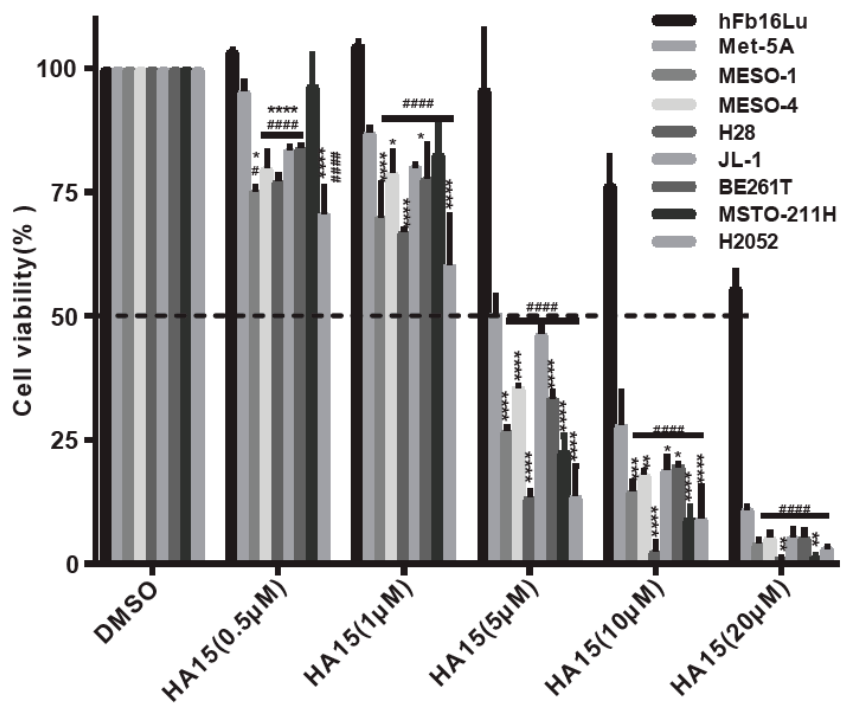

Figure 2. Excess ER stress induced by HA15 selectively inhibits MPM cells. (A,B) Cell viability and $\mathrm{IC}_{50}$ of normal human lung fibroblasts $(\mathrm{hFb} 16 \mathrm{Lu})$, normal human mesothelial (Met-5A), and MPM cells after treatment with HA15 for $72 \mathrm{~h}$. Data are presented as mean \pm s.d. A representative result is presented $(n=2)$. (C-E) Clonogenic assay of MPM cell lines $(\mathbf{C})$, primary MPM culture (BE261T) (D), normal human lung fibroblasts (hFb16Lu), and normal human mesothelial (Met-5A) after being treated with HA15. Colonies were stained and quantified (right panel) after 14 days. Representative images of three independent experiments $(n=3)$ are shown. Quantification $(\mathbf{E})$ was based on the results of $(\mathbf{C})$ and (D). Data are presented as mean \pm s.d. $(n=3) .{ }^{\#} p<0.05,{ }^{\# \# \#} p<0.0001$ by two-way ANOVA with Dunnett's multiple comparisons test, when compared with hFb16Lu. ${ }^{*} p<0.05,{ }^{* *} p<0.01$, ${ }^{* * *} p<0.005$, and ${ }^{* * * *} p<0.0001$ by two-way ANOVA with Dunnett's multiple comparisons test, when compared with Met-5A.

\subsection{Activation of Malfunctional UPR and Autophagy Underpins HA15 Effectiveness in MPM Cells}

Next, we addressed the molecular underpinnings of HA15 effects in MPM. HA15 induced persistent ER stress in MPM cells, measured by increased expression of key UPR genes [EIF2AK3 (PERK), ERN1 (IRE1 $\alpha)$, ATF4 (ATF4), XBP-1s, DDIT3 (CHOP)] at both mRNA and protein levels (Figure 3A-D). Notably, this HA15-induced increase of UPR gene expression (ATF4) did not occur in hFb16lu and Met-5A cells (Figure 3E). Moreover, HA15 treatment markedly augmented autophagic genes [ATG5 (Autophagy protein 5), ATG7 (Autophagy protein 7), BECN1 (Beclin-1), and LC3B-II] (Figure 4A-C) and induced massive cell death in MESO-1 cells, marked by the time- and dose-dependent increase of pro-apoptotic markers [BCL2L11 (BIM), BBC3 (PUMA), and cleaved caspase 7 (Cl.Caspase 7)] (Figure 4A-C). Flow cytometry-based measurement of apoptotic cell populations confirmed that the HA15 treatment resulted in MPM cell death in a dose-dependent manner (Figure 4D,E). 
A

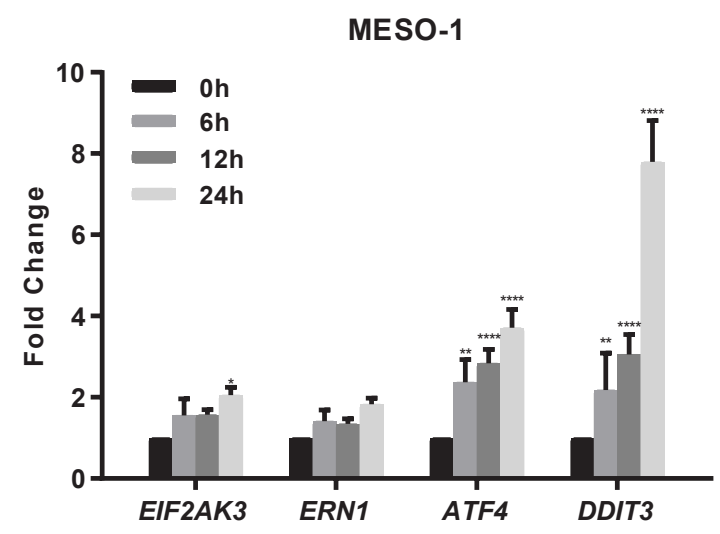

C

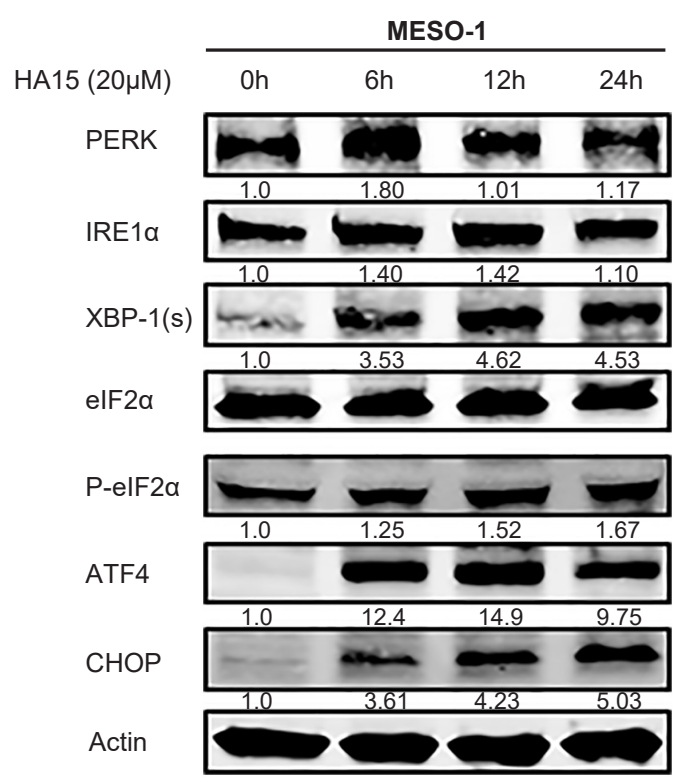

B

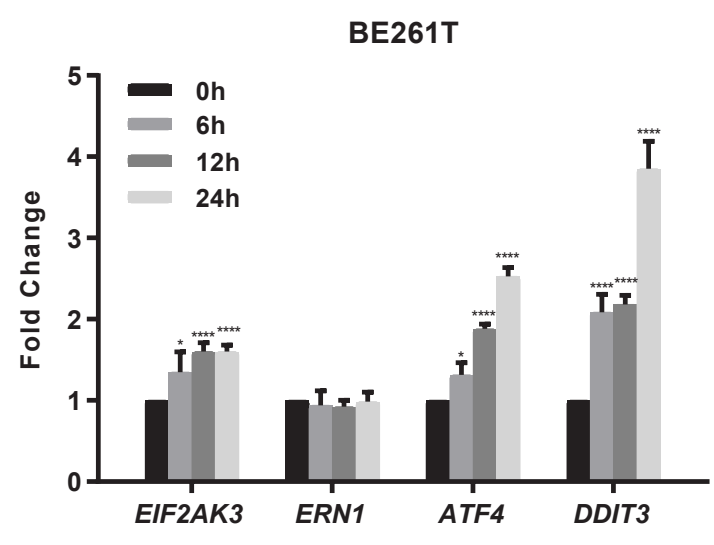

D

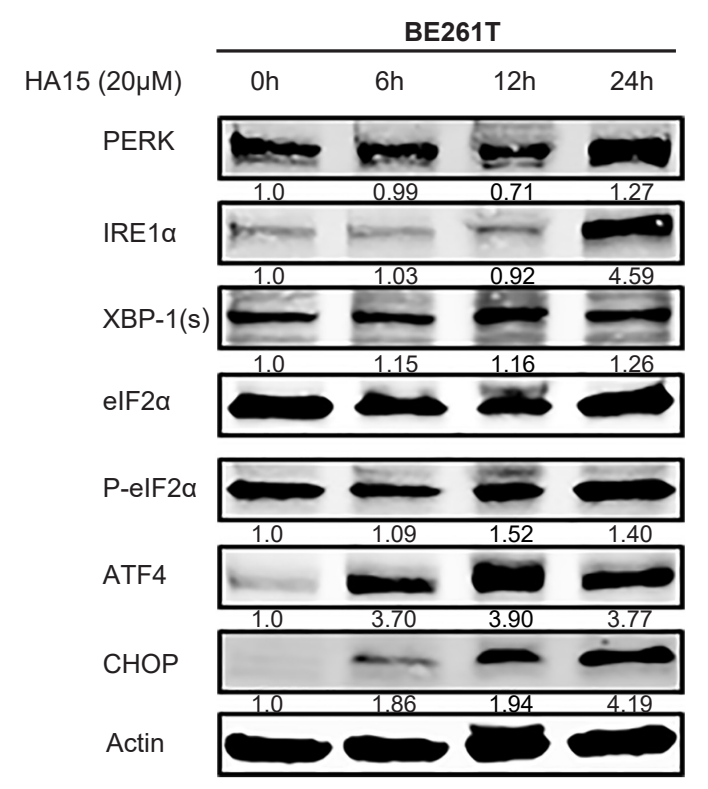

E

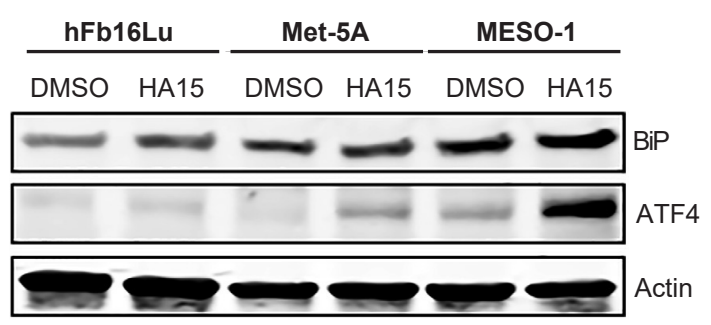

Figure 3. HA15-induced ER stress activates malfunctional UPR in MPM cells. (A,B) qRT-PCR of MESO-1 and BE261T cells after treatment with HA15 $(10 \mathrm{uM})$ for the indicated time points. Data are presented as mean \pm s.d. $(n=3) .{ }^{*} p<0.05,{ }^{* *} p<0.01$, and ${ }^{* * *} p<0.0001$ by two-way ANOVA with 
Dunnett's multiple comparisons test. (C,D) Western blots of MESO-1 and BE261T cells after treatment with HA15 $(20 \mathrm{uM})$ for the indicated time points. Quantification of the protein levels is shown under each band, with signal intensity measured by ImageJ and normalized to the loading control ( $\beta$-actin). The value of the proteins in the vehicle group was set as 1 . (E) Western blots of normal human lung fibroblasts (hFb16Lu), normal human mesothelial (Met-5A), and MPM cells (MESO-1) after treatment with HA15 (20 uM) for $24 \mathrm{~h}$. Protein quantification is shown underneath, whereby signal intensity was assessed by ImageJ and normalized to the loading control ( $\beta$-actin), with the value of the DMSO group set as 1 .

A

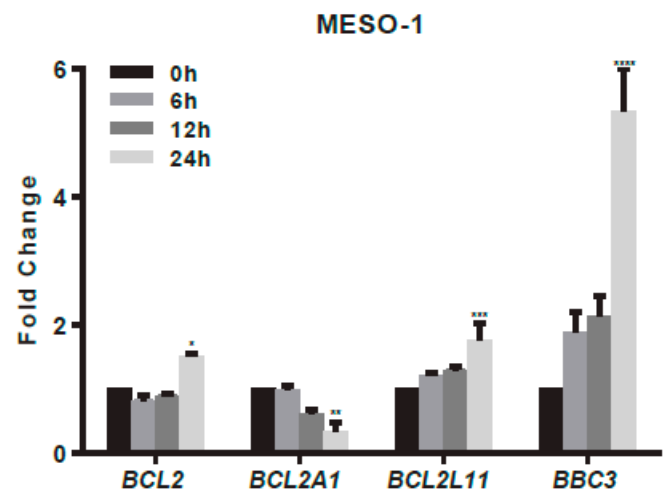

C

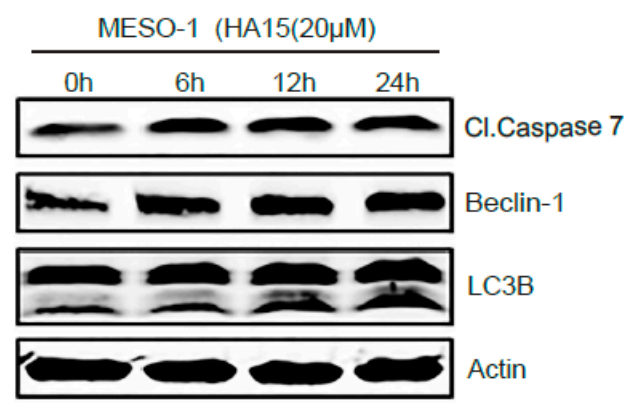

B

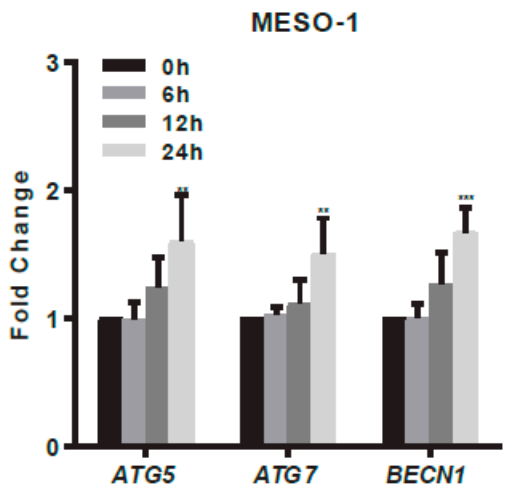

D

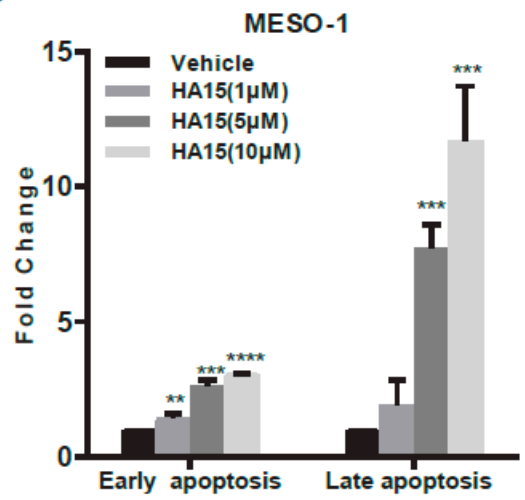

Figure 4. Cont. 
E

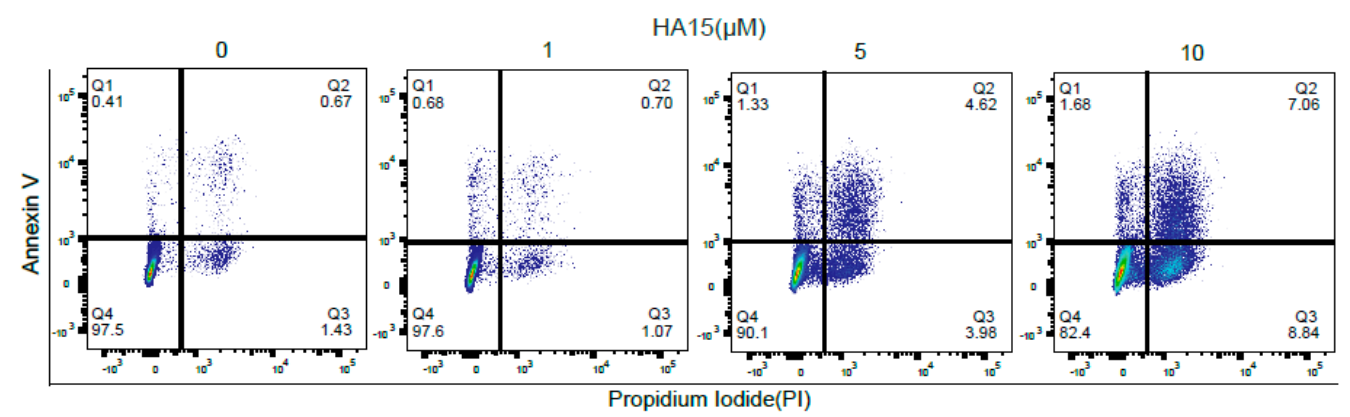

F

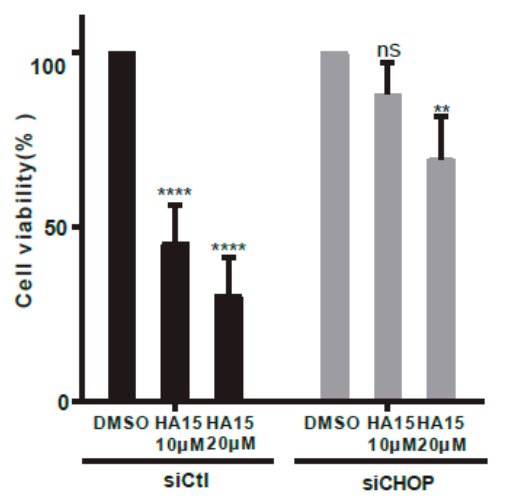

G

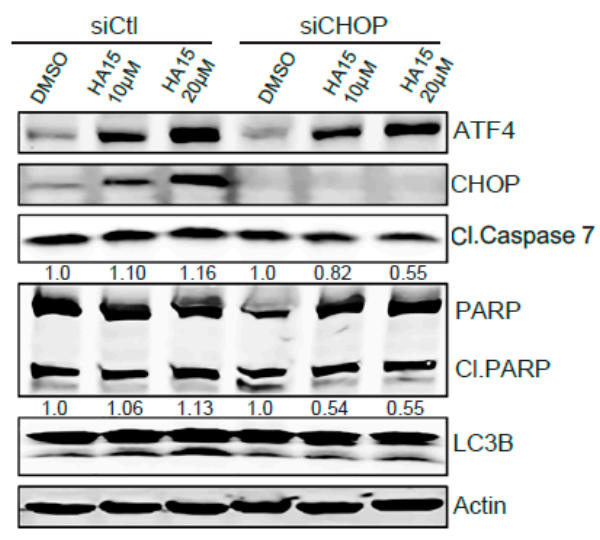

Figure 4. Activation of malfunctional UPR underpins HA15 efficacy in MPM cells. (A,B) qRT-PCR of MESO-1 cells after treated with HA15 $(10 \mathrm{uM})$ for the indicated time points. Data are presented as mean \pm s.d. $(n=3) .{ }^{*} p<0.05,{ }^{* *} p<0.01,{ }^{* * *} p<0.005$, and ${ }^{* * *} p<0.0001$ by two-way ANOVA with Dunnett's multiple comparisons test. (C) Western blots of MESO-1 cells after treatment with HA15 $(20 \mathrm{uM})$ for the indicated time points. Quantification of the protein levels is shown under each band, with signal intensity measured by ImageJ and normalized to the loading control ( $\beta$-actin). The value of the proteins in the vehicle group was set as 1. (D,E) FACS-based apoptotic assay of MESO-1 cells after treatment with indicated doses of HA15 for $72 \mathrm{~h}$. Data are presented as mean \pm s.d. $(n=3) .{ }^{* *} p<0.01$, *** $p<0.005$, and ${ }^{* * *} p<0.0001$ by two-way ANOVA with Dunnett's multiple comparisons test. A representative FACS plot is shown (E) with early and late apoptosis quantified by the Annexin $\mathrm{V}^{+} / \mathrm{PI}^{-}$ and Annexin $\mathrm{V}^{+} / \mathrm{PI}^{+}$population, respectively. (F) MESO-1 cells transfected with the control (siCtrl) or CHOP siRNAs (siCHOP) were treated with indicated doses of HA15. Cell viability was measured $48 \mathrm{~h}$ after treatment $(\mathbf{F})$. Data are presented as mean \pm s.d. $(n=3) .{ }^{* *} p<0.01$, and ${ }^{* * * *} p<0.0001$ by two-way ANOVA with Dunnett's multiple comparisons test. (G) Cell lysates were prepared from MESO-1 cells transfected with the control (siCtrl) or CHOP siRNAs (siCHOP) and analyzed by immunoblots. Protein quantification is shown underneath, whereby signal intensity was assessed by ImageJ and normalized to the loading control ( $\beta$-actin), with the value of the DMSO group set as 1 .

To test if the ER stress/UPR signaling axis is functionally important for HA15 effects on MPM cells, we knocked down DDIT3 (encoding CHOP), a key effector of malfunctional UPR that leads to apoptosis. While HA15 dose-dependently decreased cell viability and concomitantly increased CHOP, apoptosis (cleaved caspase 7/PARP), and autophagy (LC3B-II/LC3B-I ratio) in MESO-1 cells with intact CHOP (siControl), CHOP depletion significantly attenuated HA15-induced growth inhibition, apoptotic, and autophagic indices in MESO-1 cells (Figure 4F,G). As expected, ATF4, which acts upstream of CHOP, was largely unaffected by CHOP downregulation and remained active in response to HA15 (Figure 4G). These data delineate that HA15 selectively triggers MPM cell death by inducing excessive ER stress and the onset of malfunctional UPR and autophagy. 


\subsection{HA15 Potently Inhibits Chemo-Resistant MPM Cells}

To test if the effectiveness of HA15 can be extended to MPM cells resistant to standard chemotherapy, we generated chemo-resistant MPM cells by chronic exposure to stepwise-increasing doses of cisplatin/pemetrexed (Figure 5A). Both chemo-resistant (_R) and parental (_S) MPM cells responded to HA15 in a dose-dependent manner (Figure 5B,C). Coherent with the results from parental chemo-naïve MPM cells (Figure 3), HA15 induced excess ER stress, malfunctional UPR, and apoptotic cell death in chemo-resistant MPM cells, gauged by significant upregulation of UPR genes (EIF2AK3, ERN1, ATF4, DDIT3), autophagy (ATG5, BECN1), and pro-apoptosis [BCL2L11 (BIM), BBC3 (PUMA)] (Figure 5D-F). Western blot analysis confirmed that HA15 invoked ER stress-activated malfunctional UPR (increased p-eIF2 $\alpha$ and CHOP) in chemo-resistant MPM cells (Figure 5G), which is known to lead to apoptosis.

A

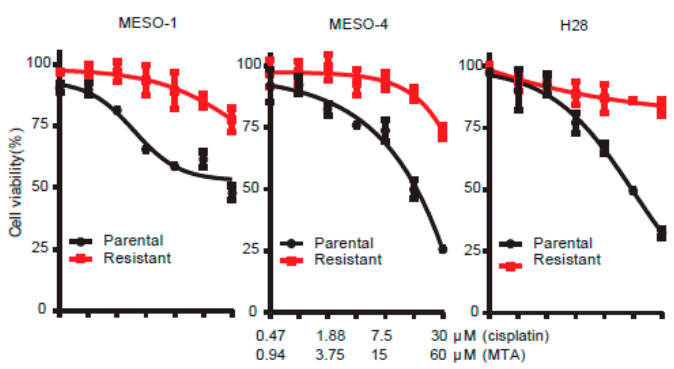

B

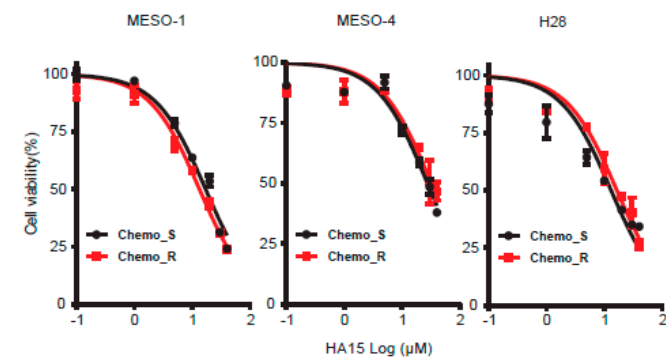

C
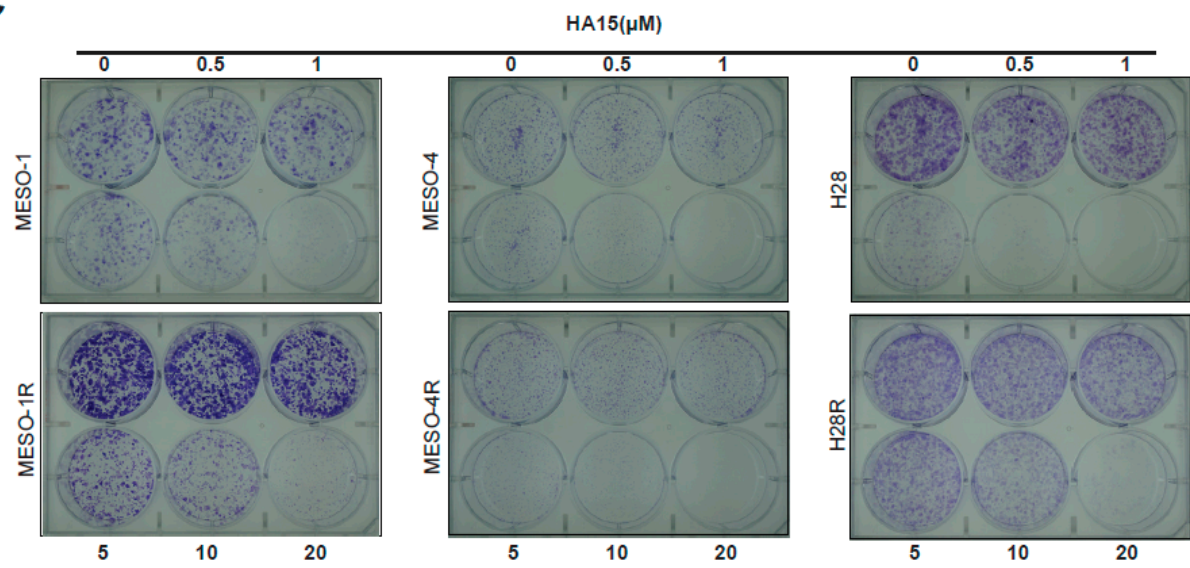

D

MESO-1(Chemo R)
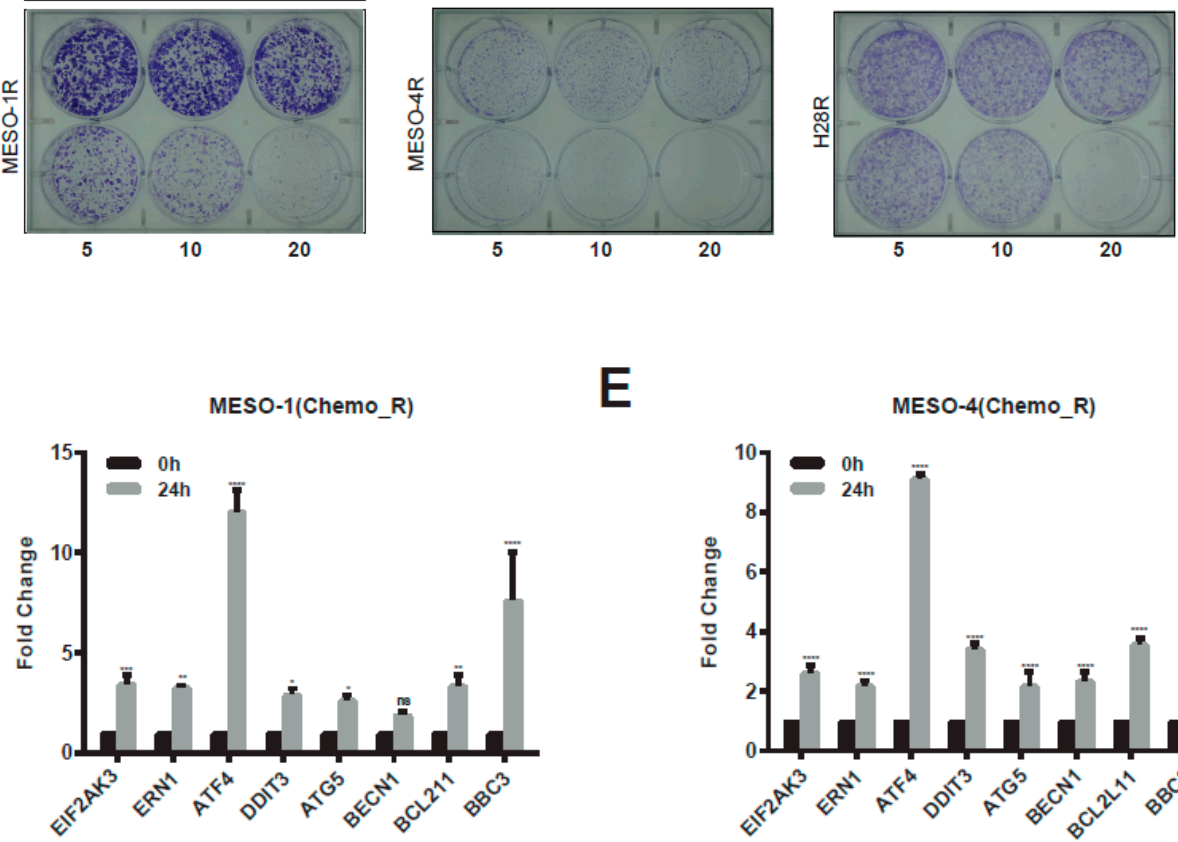

$\mathbf{E}$

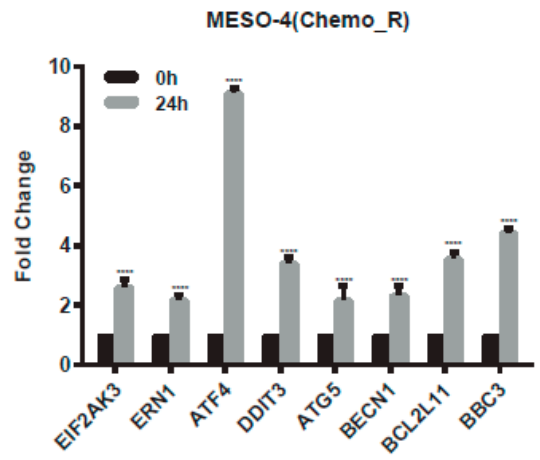

Figure 5. Cont. 
$\mathbf{F}$

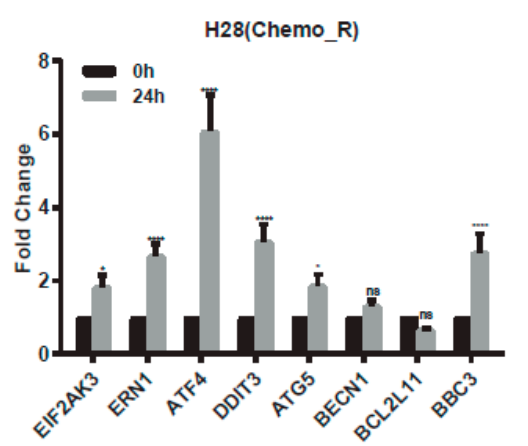

G

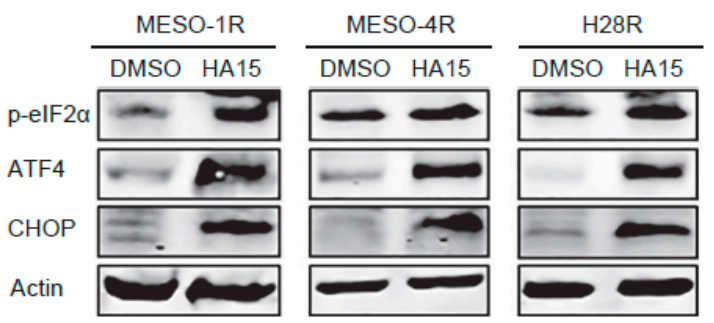

Figure 5. HA15 overcomes chemo-resistance in MPM cells. (A,B) Viability assay of parental (Chemo_S) and resistant (Chemo_R) MPM cells after treatment with cisplatin/pemetrexed or HA15 for $72 \mathrm{~h}$. Data are presented as mean \pm s.d. (A) representative result is presented $(n=2)$. (C) Clonogenic assay of parental MPM cells (MESO-1, MESO-4, H28) and resistant MPM cells (MESO-1R, MESO-4R, H28R) treated with HA15 for 14 days. Representative images of three independent experiments $(n=3)$ are shown. (D-F) qRT-PCR of resistant MPM cells after treated with HA15 $(20 \mu \mathrm{M})$ for $24 \mathrm{~h}$. Data are presented as mean \pm s.d. $(n=3) .{ }^{*} p<0.05,{ }^{* *} p<0.01, * * * p<0.005$, and ${ }^{* * * *} p<0.0001$ by two-way ANOVA with Sidak's multiple comparisons test. (G) Immunoblots of chemo-resistant MPM cells treated with HA15 $(20 \mu \mathrm{M})$ or vehicle (DMSO) for $24 \mathrm{~h}$. Protein quantification is shown underneath, whereby signal intensity was assessed by ImageJ and normalized to the loading control ( $\beta$-actin), with the value of the DMSO group set as 1 .

\subsection{HA15 Suppresses MPM Tumor Growth In Vivo}

Finally, we tested in vivo efficacy of HA15 through a comparison with standard chemotherapy (cisplatin/pemetrexed (MTA) in a patient (BE261T)-derived xenograft (PDX) model of MPM. Whereas HA15 and cisplatin/MTA alone delayed PDX tumor growth compared to the vehicle treatment, HA15 at the administrated dose exhibited greater anti-tumor effect than chemo (Figure 6A-C). Notably, the efficacy of chemo was achieved at the cost of high toxicity, but HA15 at the used dose showed no obvious side effects, monitored by body weights (Figure 6D) and a toxicity analysis that assessed liver histology and hepatic transaminase (AST/ALT) activities in the treated mice (Figure 6E,F). Importantly and in line with our in vitro results, PDX tumors after HA15 treatment displayed persistent activation of ER stress-induced UPR (PERK, IRE1 $\alpha$, p-eIF2 $\alpha$, ATF4) and autophagy (Beclin-1, LC3B-II), which was deemed anti-survival (CHOP), and enhanced cell death (Cl PARP, $\mathrm{Cl}$ Cas 7, Cl Cas 3) (Figure 6G,H). Thus, pharmacological perturbation of ER stress/UPR signaling by HA15 shows strong anti-MPM efficacy by inducing tumor cell death, validating HA15 as a potential therapeutic for MPM.

A

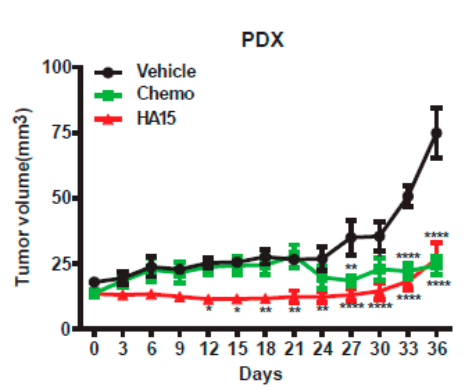

B

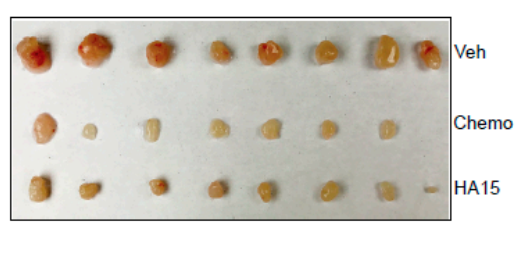

C

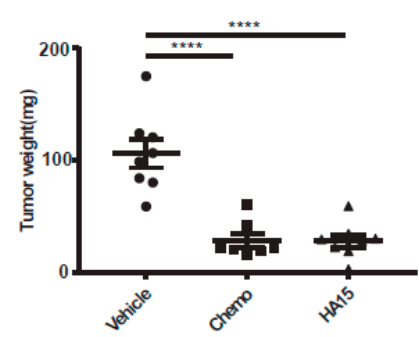

Figure 6. Cont. 


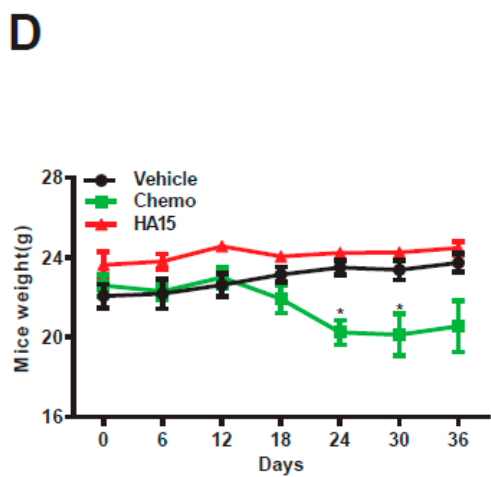

$\mathbf{E}$

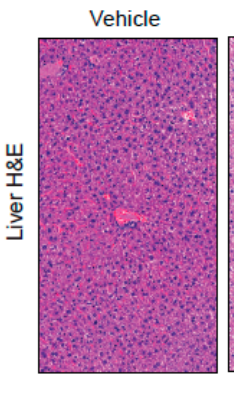

$\mathbf{F}$

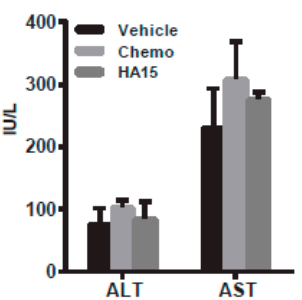

$\mathbf{G}$

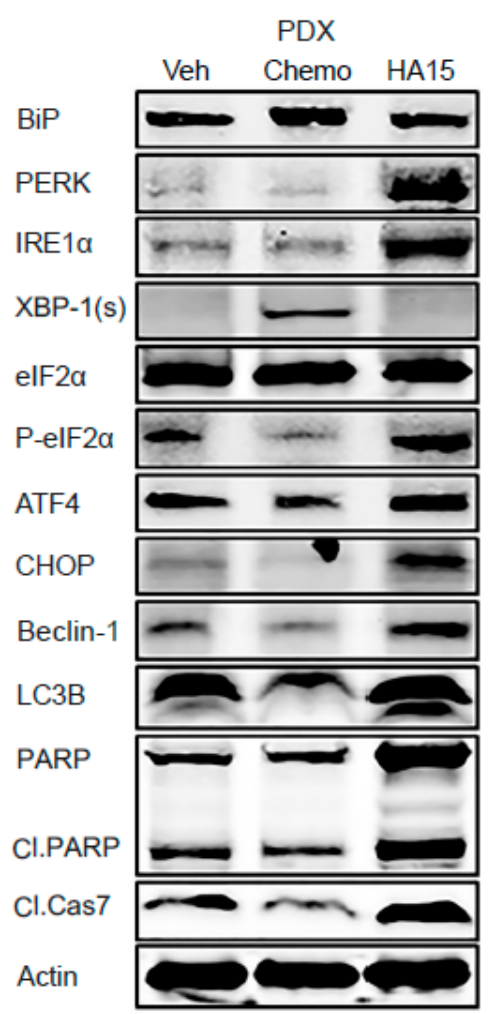

$\mathrm{H}$

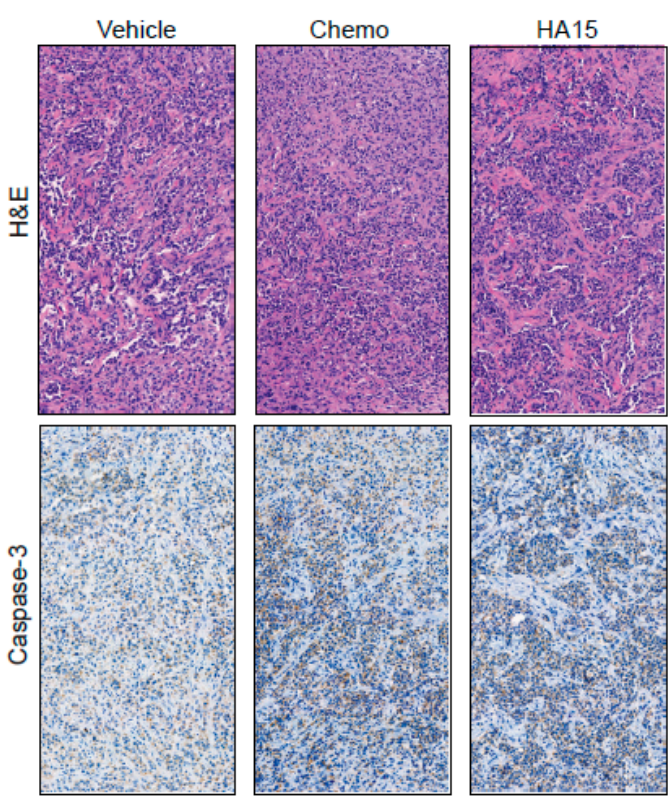

Figure 6. HA15 suppresses MPM tumor growth in vivo. (A) Growth curves of patient (BE261T)-derived xenograft (PDX) tumors treated with the vehicle, cisplatin/pemetrexed (3.75/83 mg/kg; chemo), or HA15 $(0.7 \mathrm{mg} /$ mouse $)$ for the indicated time. Data are presented as mean $\pm \operatorname{SEM}(n=4) .{ }^{*} p<0.05,{ }^{* *} p<0.01$, and ${ }^{* * * *} p<0.0001$ by two-way ANOVA with Dunnett's multiple comparisons test. (B,C) Tumor size (B) and weights (C) of PDX (BE261T) tumors after the treatment. Data are presented as mean \pm SEM $(n=4)$. ${ }^{*} p<0.05,{ }^{* *} p<0.01,{ }^{* * *} p<0.001$, and ${ }^{* * *} p<0.0001$ by one-way ANOVA with Dunnett's multiple comparisons test. (D) Mice body weights during treatment with HA15, chemo, or vehicle. Data are presented as mean $\pm \operatorname{SEM}(n=4) .{ }^{*} p<0.05$ by two-way ANOVA with Dunnett's multiple comparisons test. (E) Liver histology from treated mice. Original overall magnification, $\times 400$. (F) Transaminase (AST/ALT) activities of treated mice. Data are presented as mean $\pm \operatorname{SEM}(n=3)$. (G) Immunoblots of PDX tumors after the treatment. Protein quantification is shown underneath, whereby signal intensity was assessed by ImageJ and normalized to the loading control ( $\beta$-actin), with the value of the vehicle group set as 1. (H,E) Staining and IHC analysis for caspase-3 of PDX tumors after the treatment. Original overall magnification, $\times 400$. 


\section{Discussion}

We previously reported that dysregulated ER stress/UPR signaling is an important mechanism underlying resistance to standard cisplatin/pemetrexed chemotherapy, and that further induction of persistent ER stress overcomes chemo-resistance [26]. In this study, we discovered that deregulation of ER stress/UPR signaling is a characteristic feature for MPM, which confers a therapeutic vulnerability that normal mesothelial counterparts lack. We further demonstrated that pharmacologic augmentation of ER stress by HA15 selectively targets MPM without apparent side effects in immune-deficient NSG mice, leading to apoptotic cell death of MPM cells in vitro and potent suppression of MPM tumor growth in preclinical mouse models. Mechanistically, HA15 induces excessive proteotoxic pressures above the already high levels of ER stress in MPM, which embarks malfunctional UPR, autophagy, and eventually activates programmed cell death. These results provide a therapeutic rationale by targeting ER stress/UPR signaling in MPM and validate HA15 as a potential therapeutic for MPM.

Perturbation of ER stress/UPR can be achieved by pharmacological strategies, and small molecules that target key components of the UPR machinery (e.g., the enzymatic activity of PERK (kinase), IRE1 $\alpha$ (RNAse) and the eukaryotic initiation factor eIF2 $\alpha$ ) have been investigated in preclinical studies [33]. Although pharmacological modulation of ER stress/UPR has demonstrated significant anti-tumor activity in a variety of cancer models, undesired on-target side effects of ER stress modulators have remained incompletely understood. For instance, chronic administration of PERK inhibitors has been reported to impair pancreatic $\beta$-cells [34,35]. Furthermore, ER stress/UPR plays a key role in immune surveillance [14]. As a consequence, deregulation of the IRE1 $\alpha$-XBP1 axis has been reported to account for dysfunctional dendritic cells (DCs) and neutrophils [36], and CHOP is critical for the immune inhibitory activity of tumor-infiltrating myeloid-derived suppressor cells (MDSCs) and of $\mathrm{CD} 8^{+} \mathrm{T}$ cells $[37,38]$.

Predominantly driven by pharmacologically intractable genetic alterations in tumor suppressers [8-10], MPM remains the epitome of a lethal malignancy recalcitrant to targeted therapy efforts. By capitalizing on an integrative approach, here, we discovered that ER stress/UPR signaling is significantly deregulated in MPM compared to normal mesothelial cells. In particular, the molecular chaperon BiP, a member of the heat shock protein 70 (HSP70) family and a master regulator of ER stress response, is generally expressed at a high level in patients' MPM and established MPM cell lines. BiP cycles between the membrane and lumen of the ER, which functions as a molecular switch that senses the magnitude of ER stress and sets the threshold for the onset of ER stress-responsive UPR [39]. As the outcome of the UPR is bi-directional, pro-survival for readily relievable ER stress or pro-apoptotic if persistent and incurable ER stress emanates, targeting ER stress signaling aimed at tipping the UPR from a pro-survival to a pro-apoptotic mechanism, the latter executed by malfunctional UPR, has emerged as a promising strategy of cancer therapy [26]. As proof of our finding, MPM cells, regardless of chemotherapy-naïve or -resistant, were equally susceptible to HA15, a small molecule that induces excess ER stress by specifically targeting BiP [29]. Indeed, the antitumor effect of HA15 is intimately linked with its ability to augment UPR, as genetic depletion of CHOP, a master regulator of apoptotic cell death elicited by malfunctional UPR, abrogates HA15 effectiveness in MPM.

In summary, it remains paramount to identify new and effective therapeutic approaches to improve the clinical outcome of MPM patients. Our finding that deregulation of ER stress and the adaptive UPR sensitizes MPM cells to agents that induce excess ER stress and alter the adaptive UPR ushers in a therapeutically actionable strategy for MPM and supports further clinical investigations of HA15 to treat patients with MPM, although the potential side effects of HA15 on immunity remains to be addressed. 


\section{Materials and Methods}

\subsection{Cell Culture and Reagents}

Normal human lung fibroblasts hFb16Lu (CCD-16Lu, RRID: CVCL_2378), normal human mesothelial cells Met-5A (MeT-5A, RRID: CVCL_3749), MPM cell lines H28 (NCI-H28, RRID: CVCL_1555), H2452 (NCI-H2452, RRID: CVCL_1553), and H2052 (NCI-H2052, RRID:CVCL_1518) were obtained from ATCC (American Type Culture Collection, Manassas, VA, USA). MPM cell lines MESO-1 (ACC-MESO-1, RRID: CVCL_5113) and MESO-4 (ACC-MESO-4, RRID: CVCL_5114) were obtained from RIKEN Cell Bank (Ibaraki, Japan) [26,40-42]. MPM cell lines MSTO-211H (RRID: CVCL_1430) and JL-1 (RRID: CVCL_2080) were purchased from DSMZ (German Collection of Microorganisms and Cell Cultures, Brunswick, Germany). A primary MPM cell culture (BE261T) was established from surgically resected tumors of a 67 year-old male patient using the same protocol as described in $[26,43]$ and used for short-term studies (up to eight passages in vitro). The human study was performed under the auspices of protocols approved by institutional review board (KEK number: 042/15), and informed consent was obtained from patients. Cells were cultured in RPMI-1640 medium or Medium 199 (Cat. \#8758 and \#4540; Sigma-Aldrich, St. Louis, MO, USA) supplemented with 10\% fetal bovine serum/FBS (Cat. \#10270-106; Life Technologies, Grand Island, NY, USA) and 1\% penicillin/streptomycin solution (Cat. \#P0781, Sigma-Aldrich, St.Louis, MO, USA). All human cell lines have been authenticated using STR profiling within the last three years and are confirmed free from mycoplasma contamination (Microsynth, Bern, Switzerland). Cisplatin, pemetrexed/MTA (Cat. \#VL7640) and HA15 (Cat. \#CS-5825) were obtained from Sandoz, Eli Lilly (Vernier, Suisse) S.A. (Vernier/Geneva, Switzerland) and ChemScene (Monmouth Junction, NJ, USA), respectively.

Chemo-resistant cells (H28R, MESO-1R, and MESO-4R) were generated by chronic exposure to cisplatin/MTA following a weekly schedule of 4-day treatment and 3-day recovery. Drug treatment was initiated from cisplatin $(0.1 \mu \mathrm{M}) / \mathrm{MTA}(0.5 \mu \mathrm{M})$ and increased in a stepwise manner until cisplatin $(3 \mu \mathrm{M}) / \mathrm{MTA}(5 \mu \mathrm{M})$. Induction of resistance was measured by the cell viability assay.

\subsection{Cell Viability and Clonogenic Survival Assay}

MPM cells seeded in 96-well plates (2500 cells/well) were dosed $24 \mathrm{~h}$ later with HA15 for $72 \mathrm{~h}$. Cell viability was determined by acid phosphatase (APH) assay as described $[26,43,44]$. The efficacy of drugs on cell growth was normalized to the untreated control. Each data point was generated in triplicate and each experiment was done three times $(n=3)$. Unless otherwise stated, a representative result is presented. Best-fit curve was generated in GraphPad Prism [(log (inhibitor) vs. response (-variable slope four parameters)]. Error bars are mean \pm s.d.

Clonogenic assay was performed as described [26,43,44]. In brief, exponentially grown MPM cells seeded in 6-well plates (1000 cells/well) were dosed $24 \mathrm{~h}$ later and continually treated with HA15 for 14 days (refresh drugs every three days), the resulting colonies were stained with crystal violet $(0.5 \%$ dissolved in $25 \%$ methanol). Growth curve was generated by eluting crystal violet staining with $10 \%$ acetic acid and measuring absorbance at $590 \mathrm{~nm}$. Three independent experiments were performed.

\subsection{Quantitative Real-Time PCR ( $q$ RT-PCR)}

Total RNA was isolated and purified by RNeasy Mini Kit (Cat. \#74106; Qiagen, Germany). Complementary DNA (cDNA) was synthesized by the high capacity cDNA reverse transcription kit (Cat. \#4368814; Applied Biosystems, Foster City, CA, USA), according to the manufacturer's instructions. Real-time PCR was performed in triplicate on a 7500 Fast Real-Time PCR System (Applied Biosystems) with commercially available TaqMan 'Assay on Demand' primer/probes: EIF2AK3 (Hs00984005_m1), ERN1 (Hs00980095_m1), ATF4 (Hs00909569_g1), DDIT3 (Hs00358796_g1), BCL2 (Hs00608023_m1), BCL2A1 (Hs00187845_m1), BCL2L11 (Hs00708019_s1), BBC3 (Hs00248075_m1), ATG5 (Hs00355492_m1), ATG7 (Hs00197348_m1), and BECN1 (Hs00186838_m1). The expression of individual genes was normalized against GAPDH (Mm99999915_g1) using the ${ }^{\triangle \triangle} \mathrm{CT}$ method. 
The baseline and threshold for CT calculation were set automatically with the 7500 software v2.06 (Thermo Fisher Scientific, Waltham, MA, USA).

\subsection{Western Blot and Immunohistochemistry}

Cell lysates were prepared and Western blot analysis was performed as described $[26,43,44]$, with the exception that protease inhibitors (Cat. \#78440; Thermo Fisher Scientific, Waltham, MA, USA) were included in lysis buffer. In brief, equal amounts of protein lysates (10-25 $\mathrm{gg} / \mathrm{lane})$ were resolved by SDS-PAGE (Cat. \#4561033; Bio-Rad Laboratories, Hercules, CA, USA) and transferred onto nitrocellulose membranes (Cat. \#170-4158; Bio-Rad). Membranes were then blocked in blocking buffer (Cat. \#927-4000; Li-COR Biosciences, Bad Homburg, Germany) for $1 \mathrm{~h}$ at room temperature and incubated with appropriate primary antibodies overnight at $4{ }^{\circ} \mathrm{C}$. BiP (Cat. \#3183S), IRE1 $\alpha$ (Cat. \#3294S), PERK (Cat. \#5683S), eIF2 $\alpha$ (Cat. \#5324S), Phospho-eIF2 $\alpha$ (Ser 51) (Cat. \#3398S), ATF4 (Cat. \#11815S), Beclin-1 (Cat. \#3495S), LC3B (Cat. \#12741S), PARP (Cat. \#9532S), Cleaved-caspase 7(Cat. \#8438S) and Actin (Cat. \#3700S) from CST (Cell Signaling Technology, Leiden, Netherlands). IRDye 680LT-conjugated goat anti-mouse IgG (Cat. \#926-68020) and IRDye 800CW-conjugated goat anti-rabbit IgG (Cat. \#926-32211) from Li-COR Biosciences were used at 1:5000 dilutions. Finally, signals of membrane-bound secondary antibodies were imaged using the Odyssey Infrared Imaging System (Li-COR Biosciences).

Surgically removed xenograft tumors and liver sections were formalin-fixed and paraffinembedded (FFPE) and stained with hematoxylin and eosin (H\&E) using standard protocols. FFPE tumor blocks were sectioned at $4 \mu \mathrm{m}$, deparaffinized, rehydrated, and subsequently stained with an appropriate antibody (Caspase 3, Cat. \#9664, CST) using the automated system BOND RX (Leica Biosystems, Newcastle, UK). Visualization was performed using the Bond Polymer Refine Detection kit (Leica Biosystems) as instructed by the manufacturer. Images were acquired using PANNORAMIC ${ }^{\circledR}$ whole slide scanners and processed using Case Viewer (3DHISTECH Ltd., Budapest, Hungary).

\subsection{Apoptosis Assays}

MPM cells were treated for $72 \mathrm{~h}$ with vehicle control or HA15. After treatment, cells in the supernatant and adherent to plates were collected, washed with PBS, and pooled before suspended in $400 \mu \mathrm{L}$ binding buffer and stained with the Annexin V Apoptosis Detection Kit-FITC (Cat. \#88-8005; Thermo Fisher Scientific, Waltham, MA, USA) according to the manufacturer's instructions. Flow cytometry analysis was performed on a BD Biosciences LSRII flow cytometer. Three independent experiments were performed.

\section{6. siRNA Knockdown}

Knockdown of CHOP was achieved by specific duplex siRNAs (50 nmol/L) purchased from Origene Technologies (Cat. \#SR319903, Rockville, MD, USA). Transfection of siRNAs was performed with SiTran1.0 (Cat. \#TT300001, Origene Technologies, Rockville, MD, USA), according to the manufacturer's instructions.

\subsection{In Vivo Mouse Study}

Mouse studies were conducted in accordance with Institutional Animal Care and Ethical Committee-approved animal guidelines and protocols. All mouse experiments were performed in age- and gender-matched NSG (NOD-scid IL2R $\gamma^{\text {null }}$ ). Suspensions of tumor cells (in PBS) mixed with 1:1 with BD Matrigel Basement Membrane Matrix (Cat. \#356231; Corning Inc., Corning, NY, USA) were subcutaneously inoculated in left and right flanks (BE261T cells: $1 \times 10^{6} /$ injection). When tumors were palpable, mice were randomly assigned to treatment groups: (1) control; (2) cisplatin $(3.75 \mathrm{mg} / \mathrm{kg}$ ) plus pemetrexed (83 mg/kg) (i.p., once weekly) for five weeks; (3) HA15 (0.7 mg/mouse, i.p., 5 days/week) for five weeks. Mice weight and tumor size were measured every three days. Tumor 
size was calculated as follows: (length $\times$ width $\times$ width)/2. At the endpoint, blood samples were collected through cardiac puncta and subjected to the determination of transaminase activities.

\subsection{Public Databases (GEO, GSEA, TCGA, EMBL-EBI) and UPR Gene Signature}

Transcriptomic dataset of normal and MPM tumor samples (GSE2549 and GSE51024) were downloaded from the Gene Expression Omnibus (GEO) database [31,32] and subjected to gene set enrichment analysis (GSEA). Transcriptomic data of cancer patients were obtained from the Cancer Genome Atlas (TCGA) (https://portal.gdc.cancer.gov/projects/TCGA). The expression of key UPR genes in MPM cell lines were downloaded from the European Bioinformatics Institute of European Molecular Biology Laboratory (EMBL-EBI, http://www.ebi.ac.uk/). The UPR status of tumors was determined by the UPR gene signature, scored as the sum of the reactome unfolded protein response gene set.

\subsection{Statistical Analysis}

Statistical analyses were performed using GraphPad Prism 7.01 (GraphPad Software Inc., San Diego, CA, USA) unless otherwise indicated. All samples that met proper experimental conditions were included in the analysis and sample size was not pre-determined by statistical methods, but rather based on preliminary experiments. Group allocation was performed randomly. In all studies, data represent biological replicates $(n)$ and are depicted as mean values \pm s.d. or mean values \pm SEM as indicated in the figure legends. Comparison of mean values was conducted with unpaired, two-tailed Student's $t$-test, one-way ANOVA, or two-way ANOVA with Tukey's multiple comparisons test as indicated in the figure legends. In all analyses, $P$ values less than 0.05 were considered statistically significant. Gene expression and survival data derived from the public database as well as the correlation coefficient were analyzed using $\mathrm{R}$ (Version 3.4.3, https://cran.r-project.org/). For the survival analysis, patients were grouped by gene expression, where 'high' and 'low' expression groups were stratified by the optimal cut-off value.

\section{Conclusions}

Our study revealed that dysregulation of ER stress and adaptive UPR pathway is a characteristic feature of MPM, and supports further investigation of HA15 as a novel therapeutic for patients with MPM.

Supplementary Materials: The following are available online at http://www.mdpi.com/2072-6694/11/10/1502/s1, Figure S1: Uncropped blots from Figure 1F, Figure 3C-E, Figure 4C,G, Figures 5G and 6G. Numbers next to the blot indicate molecular weight $(\mathrm{kDa})$ of the marker.

Author Contributions: Conceptualization, D.X., R.A.S. and R.-W.P.; Funding acquisition, R.A.S. and R.-W.P.; Investigation, D.X., H.Y., Z.Y., S.B., and Y.G.; Methodology, D.X., Y.G., and S.-Q.L.; Project administration, R.A.S. and R.-W.P.; Resources, S.B., S.-Q.L., T.M.M., S.R.R.H., P.D., G.J.K., R.A.S. and R.-W.P.; Supervision, R.A.S. and R.-W.P.; Validation, D.X., H.Y., and Z.Y.; Visualization, D.X.; Writing-original draft, D.X. and R.-W.P.; Writing—review \& editing, D.X., H.Y., Z.Y., S.B., Y.G., S.-Q.L., T.M.M., S.R.R.H., P.D., G.J.K., R.A.S. and R.-W.P.

Funding: This work was supported by funds from the Swiss Cancer League (\#KFS-3772-08 2015; to R.-W.P.), Cancer League of the Canton of Bern (to R.-W.P.), the PhD fellowships from the China Scholarship Council (H.Y., Z.Y., and Y.G.)

Acknowledgments: We acknowledge. Med. vet. Judith Howard and the Central Diagnostic Laboratory (Department of Veterinary Clinical Medicine, University of Bern) for the analysis of transaminase activities, and Tumor Bank Bern (TBB) at the Institute of Pathology, University of Bern for collecting the clinically resected tumor samples from MPM patients.

Conflicts of Interest: The authors declare no conflicts of interest. 


\section{References}

1. McCambridge, A.J.; Napolitano, A.; Mansfield, A.S.; Fennell, D.A.; Sekido, Y.; Nowak, A.K.; Reungwetwattana, T.; Mao, W.; Pass, H.I.; Carbone, M.; et al. Progress in the Management of Malignant Pleural Mesothelioma in 2017. J. Thorac. Oncol. 2018, 13, 606-623. [CrossRef] [PubMed]

2. Berzenji, L.; Van Schil, P. Multimodality treatment of malignant pleural mesothelioma. F1000Research $2018,7$. [CrossRef] [PubMed]

3. Travis, W.D.; Brambilla, E.; Burke, A.P.; Marx, A.; Nicholson, A.G. (Eds.) WHO Classification of Tumours of the Lung, Pleura, Thymus and Heart, 4th ed.; International Agency for Research on Cancer: Lyon, France, 2015; Volume 7, p. 412.

4. Zhang, W.; Wu, X.; Wu, L.; Zhang, W.; Zhao, X. Advances in the diagnosis, treatment and prognosis of malignant pleural mesothelioma. Ann. Transl. Med. 2015, 3, 182. [CrossRef] [PubMed]

5. Bibby, A.C.; Tsim, S.; Kanellakis, N.; Ball, H.; Talbot, D.C.; Blyth, K.G.; Maskell, N.A.; Psallidas, I. Malignant pleural mesothelioma: An update on investigation, diagnosis and treatment. Eur. Respir. Rev. 2016, 25, 472-486. [CrossRef]

6. Linton, A.; Cheng, Y.Y.; Griggs, K.; Kirschner, M.B.; Gattani, S.; Srikaran, S.; Chuan-Hao Kao, S.; McCaughan, B.C.; Klebe, S.; van Zandwijk, N.; et al. An RNAi-based screen reveals PLK1, CDK1 and NDC80 as potential therapeutic targets in malignant pleural mesothelioma. Br. J. Cancer 2014, 110, 510-519. [CrossRef]

7. Vogelzang, N.J.; Rusthoven, J.J.; Symanowski, J.; Denham, C.; Kaukel, E.; Ruffie, P.; Gatzemeier, U.; Boyer, M.; Emri, S.; Manegold, C.; et al. Phase III study of pemetrexed in combination with cisplatin versus cisplatin alone in patients with malignant pleural mesothelioma. J. Clin. Oncol. 2003, 21, 2636-2644. [CrossRef]

8. Bueno, R.; Stawiski, E.W.; Goldstein, L.D.; Durinck, S.; De Rienzo, A.; Modrusan, Z.; Gnad, F.; Nguyen, T.T.; Jaiswal, B.S.; Chirieac, L.R.; et al. Comprehensive genomic analysis of malignant pleural mesothelioma identifies recurrent mutations, gene fusions and splicing alterations. Nat. Genet. 2016, 48, 407-416. [CrossRef]

9. Hmeljak, J.; Sanchez-Vega, F.; Hoadley, K.A.; Shih, J.; Stewart, C.; Heiman, D.; Tarpey, P.; Danilova, L.; Drill, E.; Gibb, E.A.; et al. Integrative Molecular Characterization of Malignant Pleural Mesothelioma. Cancer Discov. 2018, 8, 1548-1565. [CrossRef]

10. Guo, G.; Chmielecki, J.; Goparaju, C.; Heguy, A.; Dolgalev, I.; Carbone, M.; Seepo, S.; Meyerson, M.; Pass, H.I. Whole-exome sequencing reveals frequent genetic alterations in BAP1, NF2, CDKN2A, and CUL1 in malignant pleural mesothelioma. Cancer Res. 2015, 75, 264-269. [CrossRef]

11. Bott, M.; Brevet, M.; Taylor, B.S.; Shimizu, S.; Ito, T.; Wang, L.; Creaney, J.; Lake, R.A.; Zakowski, M.F.; Reva, B.; et al. The nuclear deubiquitinase BAP1 is commonly inactivated by somatic mutations and 3p21.1 losses in malignant pleural mesothelioma. Nat. Genet. 2011, 43, 668-672. [CrossRef]

12. Wu, L.; Dell'Anno, I.; Lapidot, M.; Sekido, Y.; Chan, M.L.; Kohno, M.; Serre-Beinier, V.; Felley-Bosco, E.; de Perrot, M. Progress of malignant mesothelioma research in basic science: A review of the 14th international conference of the international mesothelioma interest group (iMig2018). Lung Cancer 2019, 127, $138-145$. [CrossRef] [PubMed]

13. Senft, D.; Ronai, Z.E.A. Adaptive Stress Responses During Tumor Metastasis and Dormancy. Trends Cancer 2016, 2, 429-442. [CrossRef] [PubMed]

14. Cubillos-Ruiz, J.R.; Bettigole, S.E.; Glimcher, L.H. Tumorigenic and Immunosuppressive Effects of Endoplasmic Reticulum Stress in Cancer. Cell 2017, 168, 692-706. [CrossRef] [PubMed]

15. Diaz-Villanueva, J.F.; Diaz-Molina, R.; Garcia-Gonzalez, V. Protein Folding and Mechanisms of Proteostasis. Int. J. Mol. Sci. 2015, 16, 17193-17230. [CrossRef] [PubMed]

16. Yadav, R.K.; Chae, S.W.; Kim, H.R.; Chae, H.J. Endoplasmic reticulum stress and cancer. J. Cancer Prev. 2014, 19, 75-88. [CrossRef]

17. Urra, H.; Dufey, E.; Avril, T.; Chevet, E.; Hetz, C. Endoplasmic Reticulum Stress and the Hallmarks of Cancer. Trends Cancer 2016, 2, 252-262. [CrossRef]

18. Sano, R.; Reed, J.C. ER stress-induced cell death mechanisms. Biochim. Biophys. Acta 2013, 1833, 3460-3470. [CrossRef]

19. Nagelkerke, A.; Bussink, J.; Sweep, F.C.; Span, P.N. The unfolded protein response as a target for cancer therapy. Biochim. Biophys. Acta 2014, 1846, 277-284. [CrossRef] 
20. Stengel, S.; Messner, B.; Falk-Paulsen, M.; Sommer, N.; Rosenstiel, P. Regulated proteolysis as an element of ER stress and autophagy: Implications for intestinal inflammation. Biochim. Biophys. Acta Mol. Cell Res. 2017, 1864, 2183-2190. [CrossRef]

21. Hetz, C.; Saxena, S. ER stress and the unfolded protein response in neurodegeneration. Nat. Rev. Neurol. 2017, 13, 477-491. [CrossRef]

22. Amodio, G.; Moltedo, O.; Faraonio, R.; Remondelli, P. Targeting the Endoplasmic Reticulum Unfolded Protein Response to Counteract the Oxidative Stress-Induced Endothelial Dysfunction. Oxid. Med. Cell Longev. 2018, 2018, 4946289. [CrossRef] [PubMed]

23. Mohan, S.; Brown, L.; Ayyappan, P. Endoplasmic reticulum stress: A master regulator of metabolic syndrome. Eur. J. Pharmacol. 2019, 860, 172553. [CrossRef] [PubMed]

24. Logue, S.E.; Gorman, A.M. Current Concepts in ER Stress-Induced Apoptosis. J. Carcinog. Mutagen. $2013,6$. [CrossRef]

25. Xu, C.; Bailly-Maitre, B.; Reed, J.C. Endoplasmic reticulum stress: Cell life and death decisions. J. Clin. Investig. 2005, 115, 2656-2664. [CrossRef]

26. Xu, D.; Liang, S.Q.; Yang, H.; Luthi, U.; Riether, C.; Berezowska, S.; Marti, T.M.; Hall, S.R.R.; Bruggmann, R.; Kocher, G.J.; et al. Increased sensitivity to apoptosis upon endoplasmic reticulum stress-induced activation of the unfolded protein response in chemotherapy-resistant malignant pleural mesothelioma. Br. J. Cancer 2018, 119, 65-75. [CrossRef]

27. Garg, A.D.; Maes, H.; van Vliet, A.R.; Agostinis, P. Targeting the hallmarks of cancer with therapy-induced endoplasmic reticulum (ER) stress. Mol. Cell Oncol. 2015, 2, e975089. [CrossRef]

28. Xu, W.; Neckers, L. Gr(i)p the ER to Stress Out Melanoma. Cancer Cell 2016, 29, 769-771. [CrossRef]

29. Cerezo, M.; Lehraiki, A.; Millet, A.; Rouaud, F.; Plaisant, M.; Jaune, E.; Botton, T.; Ronco, C.; Abbe, P.; Amdouni, H.; et al. Compounds Triggering ER Stress Exert Anti-Melanoma Effects and Overcome BRAF Inhibitor Resistance. Cancer Cell 2016, 29, 805-819. [CrossRef]

30. Ruggiero, C.; Doghman-Bouguerra, M.; Ronco, C.; Benhida, R.; Rocchi, S.; Lalli, E. The GRP78/BiP inhibitor HA15 synergizes with mitotane action against adrenocortical carcinoma cells through convergent activation of ER stress pathways. Mol. Cell Endocrinol. 2018, 474, 57-64. [CrossRef]

31. Gordon, G.J.; Rockwell, G.N.; Jensen, R.V.; Rheinwald, J.G.; Glickman, J.N.; Aronson, J.P.; Pottorf, B.J.; Nitz, M.D.; Richards, W.G.; Sugarbaker, D.J.; et al. Identification of novel candidate oncogenes and tumor suppressors in malignant pleural mesothelioma using large-scale transcriptional profiling. Am. J. Pathol. 2005, 166, 1827-1840. [CrossRef]

32. Suraokar, M.B.; Nunez, M.I.; Diao, L.; Chow, C.W.; Kim, D.; Behrens, C.; Lin, H.; Lee, S.; Raso, G.; Moran, C.; et al. Expression profiling stratifies mesothelioma tumors and signifies deregulation of spindle checkpoint pathway and microtubule network with therapeutic implications. Ann. Oncol. 2014, 25, 1184-1192. [CrossRef] [PubMed]

33. Hetz, C.; Axten, J.M.; Patterson, J.B. Pharmacological targeting of the unfolded protein response for disease intervention. Nat. Chem. Biol. 2019, 15, 764-775. [CrossRef] [PubMed]

34. Yu, Q.; Zhao, B.; Gui, J.; Katlinski, K.V.; Brice, A.; Gao, Y.; Li, C.; Kushner, J.A.; Koumenis, C.; Diehl, J.A.; et al. Type I interferons mediate pancreatic toxicities of PERK inhibition. Proc. Natl. Acad. Sci. USA 2015, 112, 15420-15425. [CrossRef] [PubMed]

35. Dufey, E.; Sepulveda, D.; Rojas-Rivera, D.; Hetz, C. Cellular mechanisms of endoplasmic reticulum stress signaling in health and disease. 1. An overview. Am. J. Physiol. Cell Physiol. 2014, 307, C582-C594. [CrossRef] [PubMed]

36. Cubillos-Ruiz, J.R.; Silberman, P.C.; Rutkowski, M.R.; Chopra, S.; Perales-Puchalt, A.; Song, M.; Zhang, S.; Bettigole, S.E.; Gupta, D.; Holcomb, K.; et al. ER Stress Sensor XBP1 Controls Anti-tumor Immunity by Disrupting Dendritic Cell Homeostasis. Cell 2015, 161, 1527-1538. [CrossRef] [PubMed]

37. Condamine, T.; Kumar, V.; Ramachandran, I.R.; Youn, J.I.; Celis, E.; Finnberg, N.; El-Deiry, W.S.; Winograd, R.; Vonderheide, R.H.; English, N.R.; et al. ER stress regulates myeloid-derived suppressor cell fate through TRAIL-R-mediated apoptosis. J. Clin. Investig. 2014, 124, 2626-2639. [CrossRef] [PubMed]

38. Thevenot, P.T.; Sierra, R.A.; Raber, P.L.; Al-Khami, A.A.; Trillo-Tinoco, J.; Zarreii, P.; Ochoa, A.C.; Cui, Y.; Del Valle, L.; Rodriguez, P.C. The stress-response sensor chop regulates the function and accumulation of myeloid-derived suppressor cells in tumors. Immunity 2014, 41, 389-401. [CrossRef] 
39. Bertolotti, A.; Zhang, Y.; Hendershot, L.M.; Harding, H.P.; Ron, D. Dynamic interaction of BiP and ER stress transducers in the unfolded-protein response. Nat. Cell Biol. 2000, 2, 326-332. [CrossRef]

40. Usami, N.; Fukui, T.; Kondo, M.; Taniguchi, T.; Yokoyama, T.; Mori, S.; Yokoi, K.; Horio, Y.; Shimokata, K.; Sekido, Y.; et al. Establishment and characterization of four malignant pleural mesothelioma cell lines from Japanese patients. Cancer Sci. 2006, 97, 387-394. [CrossRef]

41. Cortes-Dericks, L.; Yazd, E.F.; Mowla, S.J.; Schmid, R.A.; Karoubi, G. Suppression of OCT4B enhances sensitivity of lung adenocarcinoma A549 cells to cisplatin via increased apoptosis. Anticancer Res. 2013, 33, 5365-5373.

42. Cortes-Dericks, L.; Froment, L.; Kocher, G.; Schmid, R.A. Human lung-derived mesenchymal stem cell-conditioned medium exerts in vitro antitumor effects in malignant pleural mesothelioma cell lines. Stem Cell Res. Ther. 2016, 7, 25. [CrossRef] [PubMed]

43. Liang, S.Q.; Marti, T.M.; Dorn, P.; Froment, L.; Hall, S.R.; Berezowska, S.; Kocher, G.; Schmid, R.A.; Peng, R.W. Blocking the epithelial-to-mesenchymal transition pathway abrogates resistance to anti-folate chemotherapy in lung cancer. Cell Death Dis. 2015, 6, e1824. [CrossRef] [PubMed]

44. Liang, S.Q.; Buhrer, E.D.; Berezowska, S.; Marti, T.M.; Xu, D.; Froment, L.; Yang, H.; Hall, S.R.R.; Vassella, E.; Yang, Z.; et al. mTOR mediates a mechanism of resistance to chemotherapy and defines a rational combination strategy to treat KRAS-mutant lung cancer. Oncogene 2018. [CrossRef] [PubMed]

(C) 2019 by the authors. Licensee MDPI, Basel, Switzerland. This article is an open access article distributed under the terms and conditions of the Creative Commons Attribution (CC BY) license (http://creativecommons.org/licenses/by/4.0/). 